\section{(6) OPEN ACCESS}

\begin{abstract}
- Additional material is published online only. To view please visit the journal online (http://dx.doi.org/10.1136/ gutjnl-2014-308188).

For numbered affiliations see end of article.

\section{Correspondence to} Dr Giovanni Barbara, Department of Medical and Surgical Sciences, St. OrsolaMalpighi Hospital—Building \# 5, Via Massarenti, Bologna 9-I-40138, Italy; giovanni.barbara@unibo.it
\end{abstract}

Received 2 August 2014 Revised 18 November 2014 Accepted 20 November 2014 Published Online First 22 December 2014

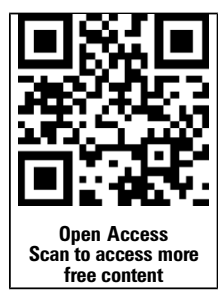

\section{SLinked}

- http://dx.doi.org/10.1136/ gutjnl-2015-309122

- http://dx.doi.org/10.1136/ gutjnl-2015-310024

\section{CrossMark}

To cite: Barbara $\mathrm{G}$, Cremon C, Annese V, et al. Gut 2016:65:82-90.

\title{
Randomised controlled trial of mesalazine in IBS
}

\author{
Giovanni Barbara, ${ }^{1}$ Cesare Cremon, ${ }^{1}$ Vito Annese, ${ }^{2}$ Guido Basilisco, ${ }^{3}$ Franco Bazzoli, ${ }^{4}$ \\ Massimo Bellini, ${ }^{5}$ Antonio Benedetti, ${ }^{6}$ Luigi Benini, ${ }^{7}$ Fabrizio Bossa, ${ }^{8}$ Paola Buldrini, ${ }^{9}$ \\ Michele Cicala, ${ }^{10}$ Rosario Cuomo, ${ }^{11}$ Bastianello Germanà, ${ }^{12}$ Paola Molteni, ${ }^{13}$ \\ Matteo Neri, ${ }^{14}$ Marcello Rodi, ${ }^{15}$ Alfredo Saggioro, ${ }^{16}$ Maria Lia Scribano, ${ }^{17}$ \\ Maurizio Vecchi, ${ }^{18}$ Giorgio Zoli, ${ }^{19}$ Roberto Corinaldesi, ${ }^{1}$ Vincenzo Stanghellini ${ }^{1}$
}

\section{ABSTRACT}

Objective Low-grade intestinal inflammation plays a role in the pathophysiology of IBS. In this trial, we aimed at evaluating the efficacy and safety of mesalazine in patients with IBS.

Design We conducted a phase 3, multicentre, tertiary setting, randomised, double-blind, placebo-controlled trial in patients with Rome III confirmed IBS. Patients were randomly assigned to either mesalazine, $800 \mathrm{mg}$, or placebo, three times daily for 12 weeks, and were followed for additional 12 weeks. The primary efficacy endpoint was satisfactory relief of abdominal pain/ discomfort for at least half of the weeks of the treatment period. The key secondary endpoint was satisfactory relief of overall IBS symptoms. Supportive analyses were also performed classifying as responders patients with a percentage of affirmative answers of at least $75 \%$ or $>75 \%$ of time.

Results A total of 185 patients with IBS were enrolled from 21 centres. For the primary endpoint, the responder patients were $68.6 \%$ in the mesalazine group versus $67.4 \%$ in the placebo group $(p=0.870 ; 95 \% \mathrm{Cl}-12.8$ to 15.1). In explorative analyses, with the $75 \%$ rule or $>75 \%$ rule, the percentage of responders was greater in the mesalazine group with a difference over placebo of $11.6 \%(p=0.115 ; 95 \% \mathrm{Cl}-2.7 \%$ to $26.0 \%)$ and $5.9 \%$ ( $p=0.404 ; 95 \% \mathrm{Cl}-7.8 \%$ to $19.4 \%$ ), respectively, although these differences were not significant. For the key secondary endpoint, overall symptoms improved in the mesalazine group and reached a significant difference of $15.1 \%$ versus placebo $(\mathrm{p}=0.032 ; 95 \% \mathrm{Cl} 1.5 \%$ to $28.7 \%$ ) with the $>75 \%$ rule.

Conclusions Mesalazine treatment was not superior than placebo on the study primary endpoint. However, a subgroup of patients with IBS showed a sustained therapy response and benefits from a mesalazine therapy.

Trial registration number ClincialTrials.gov number, NCT00626288.

\section{INTRODUCTION}

IBS is the most common functional GI disorder in which abdominal pain and/or discomfort is associated with changes in bowel habit, and with features of disordered defecation. IBS affects $10-20 \%$ of the population and causes a marked reduction of quality of life in affected individuals. ${ }^{1}$ IBS is a heterogeneous disorder involving a combination of negative life experiences and trauma, particularly early in life, genetic predisposition and environmental factors.

\section{Significance of this study}

What is already known on this subject?

- A high proportion of patients with IBS show low-grade inflammation in the intestinal mucosa.

- Low-grade inflammation is likely to be multifactorial, involving genetic factors, stress, atopy, previous episodes of infectious gastroenteritis, changes in gut microbiota and epithelial barrier defects.

- A previous proof-of-concept trial showed that mesalazine reduced the number of total immune cells and mast cells in the colonic mucosa of patients with IBS.

What are the new findings?

- This is the first large randomised controlled trial assessing the efficacy and safety of mesalazine in IBS.

- Mesalazine did not improve the percentage of patients with satisfactory relief of abdominal pain/discomfort for at least half of the weeks of the treatment period.

- Mesalazine significantly improved the percentage of patients with satisfactory relief of overall IBS symptoms if sustained responses were required ( $>75 \%$ of treatment period).

How might it impact on clinical practice in the foreseeable future?

- A subgroup of patients with IBS showed a sustained therapy response and benefits from mesalazine therapy.

Patients with IBS show dysfunction in the brain-gut axis, including anxiety, depression, gut dysmotility and visceral hypersensitivity. The relative importance of central versus peripheral mechanism in the IBS pathophysiology remains poorly defined. ${ }^{2}$ Mounting evidence is showing microscopic and molecular abnormalities in large subsets of patients with IBS. ${ }^{3}$ The most compelling evidence linking inflammation to IBS is the demonstration of the development of IBS in $3.7-36.8 \%$ of subjects following acute infectious gastroenteritis. ${ }^{4}{ }^{5}$ Mucosal and systemic immune activation have also been widely documented in patients with IBS in the absence of a previous major gastroenteritis event. ${ }^{3}$ Mucosal inflammation is linked to increased mucosal permeability, ${ }^{6}$ 
enterochromaffin cell hyperplasia and higher tissue availability of serotonin, a key factor involved in the control of gut sensorimotor functions. ${ }^{7}$ Another key finding linking inflammation to IBS pathophysiology and symptom generation is the observation that adoptive transfer of mucosal biopsy supernatants evoked activation of sensory pain pathways ${ }^{9}{ }^{10}$ and abnormal enteric nervous system responses in recipient rodents. ${ }^{11-13}$ Interestingly, these responses were reduced to a large extent by antagonism of immune-related factors including proteases and histamine. ${ }^{9} 101213$ The origin of low-grade inflammation in patients with IBS remains undetermined, but it is likely to be multifactorial, involving genetic predisposition, ${ }^{14}{ }^{15}$ stress, ${ }^{16}$ atopy, ${ }^{17}$ abnormal intestinal microbiota ${ }^{18}$ and higher mucosal permeability increasing antigenic exposure of the mucosa to luminal antigens. ${ }^{6}$

Taken together, the above-reported evidences provide the rationale to test the efficacy of intestinal anti-inflammatory compounds in patients with IBS. In a previous randomised, placebocontrolled proof-of-concept trial, we showed that compared with placebo, mesalazine reduced the number of total immune cells, T cells and mast cells in the colonic mucosa of patients with IBS. ${ }^{19}$ Although this trial was underpowered to detect effects on symptoms, a promising improvement on the patient's general well-being was identified. ${ }^{19}$ In addition, while corticosteroid treatment was ineffective in symptom improvement at least in 29 patients with postinfectious IBS, ${ }^{20}$ mast cell stabilisers, such as cromolyn sodium ${ }^{21}$ and ketotifen, ${ }^{22}$ showed promising results particularly in IBS with diarrhoea, suggesting a role of immune mechanisms and mast cells in IBS symptom generation. However, well-designed controlled studies are needed to confirm the efficacy of these drugs in IBS. Finally, mesalazine is the therapy of first choice for the treatment (clinical improvement, not necessarily remission) and maintenance of clinical remission in patients with mild-to-moderate UC. ${ }^{23}$

In the present study, we assessed the clinical efficacy and safety of mesalazine against placebo in a large, phase 3, randomised, double-blind, placebo-controlled, multicentre study in patients with IBS.

\section{METHODS}

\section{Study design}

This is a phase III, randomised, double-blind, placebocontrolled, parallel-arm, multicentre trial designed to study the efficacy and safety of mesalazine in adult patients with IBS. The study included a 2 -week screening period, a 12-week placebocontrolled treatment period and a 12-week follow-up period (figure 1). After the screening phase, eligible patients were randomly assigned to either mesalazine $800 \mathrm{mg}$ three times daily or

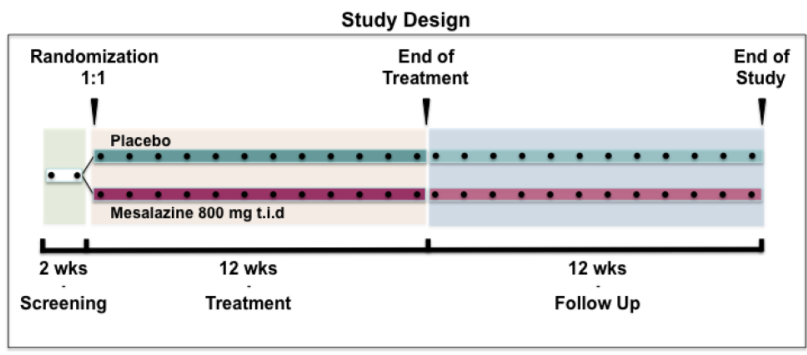

Figure 1 Study design. There was a screening period of 2 weeks before randomisation (1:1), a 12-week placebo-controlled treatment period and a 12-week follow-up period. Study visits occurred every two weeks during treatment period and every four weeks during follow-up. placebo in a 1:1 ratio. All the subjects were blindly allocated by means of scratch cards to one of the two treatment groups (mesalazine or placebo) according to a computer-generated randomisation list provided by the sponsor. A validated SAS program was used by an independent statistician to generate a randomisation list with blocks, block size $=4$, preallocated to centres. All patients, study investigators and sponsor staff were blinded to the randomisation codes. The codes were kept confidential until the end of the study when the randomisation code was broken after the database lock. Study visits were conducted every two weeks during the treatment period and every four weeks during the follow-up period.

The protocol was designed by the coordinating centre (GB, CC, RC and VS). Data were collected by investigators at each of the 21 Italian test centres and were monitored by the sponsor with the supervision of On Pharmaceutical Industry Service (OPIS) (a contract research organisation). OPIS personnel, in collaboration with the coordinating centre, analysed the trial data. A statistical analysis plan (SAP) was released and approved by the sponsor prior to the database lock and the unblinding of the treatment.

\section{Study patients}

Eligible patients with symptoms meeting Rome III criteria for diagnosis of IBS ${ }^{1}$ were recruited in the study from a total of 21 Italian centres. Inclusion and exclusion criteria are reported in online supplementary file.

\section{Study assessment}

Visits took place at screening, at baseline (randomisation visit) and every two weeks during the treatment period. During the follow-up, visits occurred monthly. The study data collection was carried out through a paper clinical case report form. Patients recorded daily all symptoms in a paper patient diary. Use of concomitant medication and adverse events were recorded at each visit. Additional safety data, such as haematology, blood chemistry and vital signs, were monitored.

The primary endpoint was assessed using a binary scale based on the patient answers to the following weekly question: "Did you have satisfactory relief of your abdominal discomfort or pain during the last week?" The patients were classified as responders if they reported an affirmative answer in at least $50 \%$ of weeks over a 3 -month treatment period $(50 \%$ rule). Explorative analyses were also performed. Patients were classified as responders if they reported a percentage of affirmative answers for at least $75 \%$ or $>75 \%$ of the treatment time $(75 \%$ rule and $>75 \%$ rule, respectively). The $75 \%$ rule was preplanned, as documented in the SAP, while the $>75 \%$ rule was post hoc and performed with the intention of minimising placebo effect and optimising drug-placebo differences.

The key secondary endpoint was assessed using a binary scale based on the patient answers to the following weekly question: "Did you have satisfactory relief of your overall IBS symptoms during the last week?" The patients were classified as responders if they reported an affirmative answer in at least $50 \%$ of weeks over a 3-month treatment period. Explorative analyses were also performed. Patients were classified as responders if they reported a percentage of affirmative answers for at least $75 \%$ or $>75 \%$ of the treatment time ( $75 \%$ rule and $>75 \%$ rule, respectively).

The other secondary endpoints included daily assessment of abdominal pain or discomfort, bloating and general well-being, monitored using a 10-point visual analogue scale, and daily assessment of stool frequency and consistency (by means of the Bristol Stool Scale Form). At the end of the treatment period, 
patients were asked to assess their overall satisfaction with treatment using a 10-point visual analogue scale. Weekly number of rescue medications was also computed. In addition, patients completed the IBS-specific quality of life questionnaire (IBS-QoL) $^{24}$ and the short-form 36 items health survey $(\mathrm{SF}-36)^{25}$ at baseline, at the end of the treatment period and at the end of follow-up.

\section{Statistical analysis}

Based on our previous proof-of-concept study, ${ }^{19}$ we estimated the sample size for the study assuming that $30 \%$ of the patients in the placebo group and $55 \%$ in the mesalazine group would meet the criteria for the primary endpoint, namely the percentage of responder patients. A sample of 136 patients (68 patients in each of the two treatment group) was needed to have $80 \%$ power to show the 25 -percentage-point difference between the groups at a significance level of 0.05 . Assuming a dropout rate of $23 \%(36 / 159)$, the sample size was adjusted to 178 patients according to Freedman's formula.

All subjects who took at least one dose of the study treatments were included in the safety population. All randomised patients who took at least one dose of the study drug and with at least one evaluation of the primary endpoint were included in the intention-to-treat (ITT) population. All randomised patients who completed the entire treatment period without any major deviation from the protocol procedures were included in the per protocol (PP) population.

The primary efficacy population was ITT. The analysis on PP population was performed for supportive data. The results of the two analyses were evaluated for consistency. The percentage of responder patients was calculated by treatment group, and the difference between the percentages was tested by means of a $\chi^{2}$ test. In order to evaluate the effect of treatment over time, a logistic model for repeated measures was applied. Dropout patients were handled in the following way: if the reason for discontinuation was the inefficacy of the therapy, patients were considered as non-responders; for all other reasons, in case of early discontinuation, a prevalence approach was adopted. In this approach, incomplete data were ignored. To illustrate the robustness of the conclusions, a sensitivity analysis was performed. The best scenario and the worst scenario were assumed for patients with missing values. In the first case, missing values were replaced with affirmative answers, in the latter with negative answers. The analysis was also performed adopting the complete case approach, considering only patients without missing data. Results were compared for consistency and if they led to similar conclusions there was reasonable assurance that the loss of information had no effect on the overall study conclusions. The CochranMantel-Haenszel test was applied to test the difference in proportion between treatment groups, adjusting for site and for IBS type. The Breslow-Day test was applied in order to evaluate the homogeneity in the OR among sites and IBS types.

All the secondary efficacy analyses were performed on the ITT population. For the key secondary endpoint, that is, the percentage of patients with satisfactory relief of overall IBS symptoms, the same approach adopted for the primary endpoint was applied. To evaluate the difference between treatments in the overall satisfaction with treatment at the end of the treatment period, the unpaired t test or the Mann-Whitney test was applied, according to the data distribution. To evaluate the difference between treatments in abdominal pain/discomfort, bloating and general well-being intensity, the analysis of covariance (ANCOVA) model for repeated measures was applied considering values at week 2 and at week 1, treatment week and the interaction treatment week as effect. Normality was assessed by means of the Shapiro-Wilk test. In case of non-normality of data, a rank transformation was applied. Stool frequency and consistency was described by treatment group and by day. Summary descriptive statistics of weekly stool frequency were provided by treatment group and by week. The weekly number of rescue medications was computed and described with summary statistics by treatment group and by week. IBS-QoL and SF-36 questionnaire data recorded at baseline, at the end of treatment and at the end of follow-up were described by treatment. The difference in answer distribution between treatments was tested by means of a Cochran-Armitage test for trend (exact version in case of cell numerousness lower than 5). No correction for multiple comparisons was applied due to the exploratory nature of this analysis. To evaluate the difference between treatments in total score and subscores, the ANCOVA model for repeated measures was applied considering value at baseline, treatment, time point and interaction treatment-time point as effect.

All statistical tests were performed with a significance level of $\alpha=0.05$. The data were analysed by means of the SAS System for Windows V.9.2.

\section{RESULTS}

\section{Study patients}

The flow chart of the enrolment and randomisation of the study is reported in figure 2. The study was conducted from February 2008 through March 2012. A total of 185 patients with IBS were included in the study. Among the enrolled patients, five were not randomised and were excluded from all analyses. In total, 180 patients were randomised, 88 allocated to mesalazine and 92 to placebo treatment groups. One patient randomised to the placebo group did not take at least one dose of the study treatment. Thus, the safety population consisted of 179 patients. Seven other patients were excluded from the ITT population as they did not have any evaluation of the primary endpoint. Thus, 172 subjects received at least one dose of the study drug and were included in the ITT population. Among the 180 randomised patients, 50 patients prematurely discontinued the treatment. The primary reason included consent withdrawal (17 patients), adverse events (14 patients), lost during follow-up (8 patients), protocol violation (6 patients) and other reasons (5 patients). Protocol violations are reported in the online supplementary file. Overall, $85.5 \%$ of patients in the safety population took the study treatments, with compliance between $70 \%$ and $120 \%$. Demographic and baseline characteristics of the safety population were similar between treatment groups and are reported in table 1.

\section{Primary efficacy analysis}

Satisfactory relief of abdominal discomfort or pain

The logistic model for repeated measures did not reveal a statistically significant effect for treatment $(p=0.324)$, nor interaction between treatment and time $(p=0.897)$. The answers varied significantly with time $(\mathrm{p}<0.001)$. Using the same model to test the simple main effect, no statistical significance was detected at any week of treatment, although a borderline effect $(\mathrm{p}=0.060)$ resulted for week 8 (figure $3 \mathrm{~A}$ ).

According to the prevalence approach, responder patients were $68.6 \%$ in the mesalazine group versus $67.4 \%$ in the placebo group, with a $\delta$ difference in favour of the mesalazine group of $1.2 \%(\mathrm{p}=0.870 ; 95 \% \mathrm{CI}-12.8 \%$ to $15.1 \%)$ (figure $3 \mathrm{~B}$ ). Non-statistically significant results were confirmed in the PP population, in which responder patients were $75.0 \%$ in the 


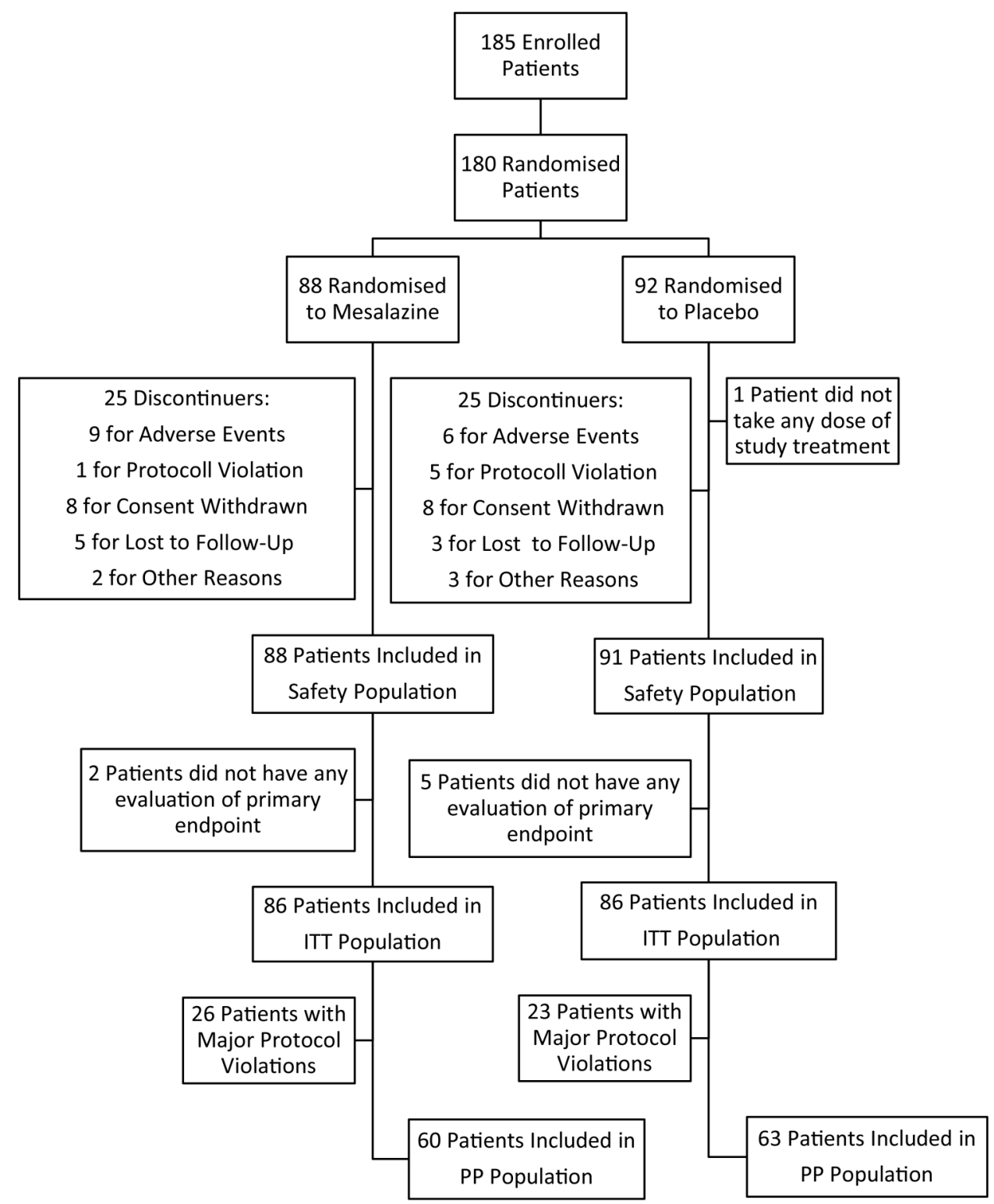

Figure 2 Flow chart of enrolment and randomisation of the study. A total of 185 patients with IBS were included in the study. Of these, five were not randomised and were excluded from all analyses. Of the 180 patients randomised, 88 were allocated to mesalazine and 92 to placebo. One patient randomised to placebo did not take at least one dose of the study treatment. Seven other patients were excluded from the intention-to-treat (ITT) population as they did not have any evaluation of the primary endpoint. Thus, 172 subjects were included in the ITT population. PP, per protocol.

mesalazine group versus $76.2 \%$ in the placebo group $(\mathrm{p}=0.878$; difference $=-1.2 \% ; 95 \% \mathrm{CI}-16.4 \%$ to $14.0 \%)$. Among patients without missing data, responder patients were $79.1 \%$ in the mesalazine group versus $78.3 \%$ in the placebo group $(\mathrm{p}=0.904$; difference $=0.8 \% ; 95 \%$ CI $-12.9 \%$ to $14.6 \%)$. In the 'worst scenario' and 'best scenario', the results were similar, indicating that missing data had no impact on the main result. The Breslow-Day test showed no homogeneity differences in the OR among sites $(p=0.464)$ and IBS subtypes (ie, IBS with constipation (IBS-C), IBS with diarrhoea (IBS-D), mixed IBS (IBS-M); $p=0.193)$. The percentage of responder patients according IBS subtypes is reported in the online supplementary file.

\section{Explorative analysis}

Figure $3 \mathrm{~B}$ shows the supportive analysis performed considering patients as responders if they reported a percentage of affirmative answers in at least $75 \%$ or $>75 \%$ of the 3 months of treatment, adopting a prevalence approach. With the $75 \%$ rule, responder patients were $43.0 \%$ in the mesalazine group versus $31.4 \%$ in the placebo group, with a $\delta$ difference of $11.6 \%$ $(\mathrm{p}=0.115 ; 95 \% \mathrm{CI}-2.7 \%$ to $26.0 \%)$. With the $>75 \%$ rule, responder patients were $32.6 \%$ in the mesalazine group versus $26.7 \%$ of patients in the placebo group, with a $\delta$ difference of $5.9 \%(\mathrm{p}=0.404 ; 95 \% \mathrm{CI}-7.8 \%$ to $19.4 \%)$.

Considering treatment and follow-up period together, no significant differences in the responder rate were observed between the mesalazine and placebo groups $(67.4 \%$ vs $61.6 \%$, respectively; $\mathrm{p}=0.426$; difference $=5.8 \% ; 95 \% \mathrm{CI}-8.46 \%$ to $20.09 \%$ ).

\section{Secondary efficacy analysis}

Satisfactory relief of overall IBS symptoms

The logistic model for repeated measures did not reveal statistically significant effects for treatment $(p=0.155)$, nor interaction between treatment and time $(p=0.640)$. The answers varied significantly with time $(p=0.004)$. Using the same model to test the simple main effect, a borderline significant effect was 
Table 1 Baseline characteristics of study participants

\begin{tabular}{|c|c|c|c|}
\hline Characteristics & $\begin{array}{l}\text { Placebo } \\
(n=91)\end{array}$ & $\begin{array}{l}\text { Mesalazine } \\
(n=88)\end{array}$ & p Value \\
\hline Age, years $( \pm S D)$ & $40.3(12.2)$ & $41.0(11.6)$ & 0.556 \\
\hline Female gender, n (\%) & $54(59.3)$ & $51(58.0)$ & 0.851 \\
\hline Ethnic origin & & & 0.613 \\
\hline Caucasian, n (\%) & $89(97.8)$ & 85 (96.6) & \\
\hline African, n (\%) & $1(1.1)$ & $2(2.3)$ & \\
\hline Asian, $\mathrm{n}(\%)$ & $1(1.1)$ & 0 & \\
\hline Other, n (\%) & $0(0)$ & $1(1.1)$ & \\
\hline IBS subtype (3) & & & 0.481 \\
\hline IBS-D, n (\%) & $39(42.8)$ & $30(34.1)$ & \\
\hline IBS-C, n (\%) & $18(19.8)$ & $18(20.4)$ & \\
\hline IBS-M, n (\%) & $34(37.4)$ & $40(45.5)$ & \\
\hline Abdominal pain score ${ }^{\star}+( \pm S D)$ & $4.5(2.3)$ & $4.6(2.5)$ & 0.930 \\
\hline Bloating score ${ }^{*} \dagger( \pm S D)$ & $5.0(2.7)$ & $5.4(2.8)$ & 0.377 \\
\hline IBS-QoLt $( \pm$ SD $)$ & $65.2(16.4)$ & $62.9(17.4)$ & 0.439 \\
\hline General well-being ${ }^{*} \dagger( \pm S D)$ & $4.1(2.5)$ & $4.8(2.5)$ & 0.074 \\
\hline SF-36† ( $( \pm \mathrm{SD})$ & $65.1(15.4)$ & $62.7(16.0)$ & 0.324 \\
\hline
\end{tabular}

detected at week $3(\mathrm{p}=0.060)$ and a statistical significance was detected at week $5(\mathrm{p}=0.038)$ (figure $4 \mathrm{~A})$.

According to the prevalence approach, responder patients were $66.3 \%$ in the mesalazine group versus $61.6 \%$ in the placebo group, with a $\delta$ in favour of the mesalazine group of $4.7 \%$ $(\mathrm{p}=0.525 ; 95 \%$ CI $-9.7 \%$ to $19.0 \%$ ) (figure $4 \mathrm{~B}$ ). Among patients without missing data, responder patients were $73.1 \%$ in the mesalazine group versus $71.0 \%$ in the placebo group $(\mathrm{p}=0.783$; difference $=2.2 \%$; $95 \%$ CI $-13.0 \%$ to $17.2 \%)$. Similar data were obtained with both the 'worst scenario' and 'best scenario', indicating that missing data had no impact on the results. The Cochran-Mantel-Haenszel detected no statistically significant difference in proportion between treatment groups adjusted for site $(p=0.513)$. The percentage of responder patients according to IBS subtypes is reported in the online supplementary file.

\section{Explorative analysis}

Figure 4B shows the supportive analysis adopting a prevalence approach. With the $75 \%$ rule, $46.5 \%$ of patients in the mesalazine group were responders versus $34.9 \%$ in the placebo group, with a $\delta$ difference of $11.6 \%(\mathrm{p}=0.121 ; 95 \%$ CI $-3.0 \%$ to $26.2 \%)$. With the $>75 \%$ rule, $38.4 \%$ of patients were responders in the mesalazine group versus $23.3 \%$ in the placebo group, with a $\delta$ difference of $15.1 \%(\mathrm{p}=0.032 ; 95 \%$ CI $1.5 \%$ to $28.7 \%$ ).

Considering treatment and the follow-up period together, no significant differences in the responder rate were observed between the mesalazine and placebo groups $(69.8 \%$ vs $59.3 \%$, respectively; $\mathrm{p}=0.151$; difference $=10.5 \%$; $95 \% \mathrm{CI}-3.8 \%$ to $24.7 \%)$. With the $75 \%$ rule, $41.9 \%$ of patients were responders in the mesalazine group versus $29.1 \%$ in the placebo group. The difference was $12.8 \%$ and borderline significant $(\mathrm{p}=0.080$; difference $=12.8 \%$; $95 \% \mathrm{CI}-1.4 \%$ to $27.0 \%$ ).

\section{Abdominal pain/discomfort, bloating and stool characteristics}

Table 2 reports descriptive statistics by treatment group and by week for abdominal pain/discomfort and bloating. The ANCOVA model for repeated measures applied to the changes versus values at week 2 did not detect a statistically significant
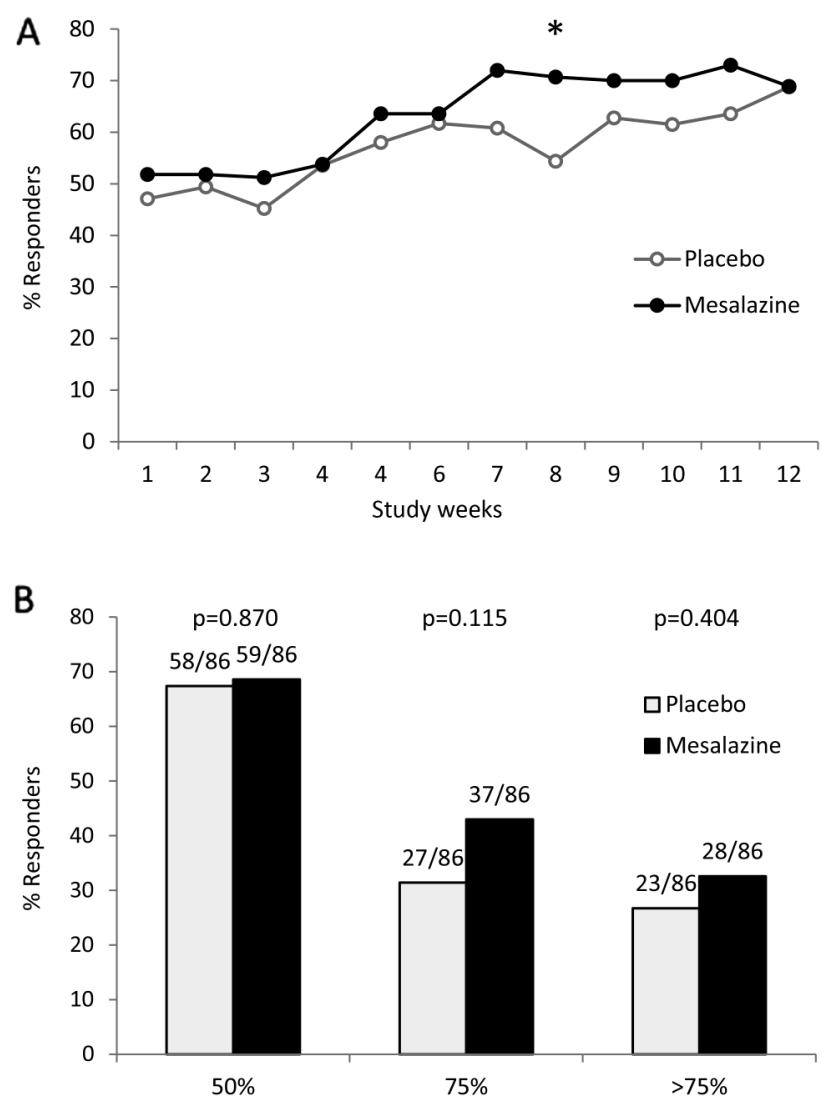

Figure 3 Primary efficacy analysis (satisfactory relief of abdominal pain or discomfort). The logistic model for repeated measures did not reveal a statistically significant effect for treatment $(p=0.324)$ nor interaction between treatment and time $(p=0.897)$. The answers varied significantly with time $(p<0.001)$. Using the same model to test the simple main effect, a borderline significant effect $(p=0.060)$ resulted for week 8 (A). According to the prevalence approach, responder patients were $68.6 \%$ in the mesalazine group versus $67.4 \%$ in the placebo group, with a $\delta$ difference in favour of the mesalazine group of $1.2 \%(p=0.870 ; 95 \% \mathrm{Cl}-12.8 \%$ to $15.1 \%)$ (B). Explorative analyses. With the $75 \%$ rule, $43.0 \%$ of patients in the mesalazine group were responders versus $31.4 \%$ in the placebo group, with a $\delta$ difference of $11.6 \%(p=0.115 ; 95 \% \mathrm{Cl}-2.7 \%$ to $26.0 \%)(\mathrm{B})$. With the $>75 \%$ rule, $32.6 \%$ of responder patients were identified in the mesalazine group versus $26.7 \%$ of patients in the placebo group, with a $\delta$ difference of $5.9 \%(p=0.404 ; 95 \% \mathrm{Cl}-7.8 \%$ to $19.4 \%)(B)$.

difference between treatment groups for all the aforementioned analyses (abdominal pain/discomfort: $p=0.903$; bloating: $\mathrm{p}=0.184$ ). No relevant difference between treatment groups was detected for stool consistency and stool frequency (see online supplementary file).

General well-being, IBS-QoL, SF-36 and satisfaction with treatment

Table 2 reports descriptive statistics by treatment group and by week for general well-being, IBS-QoL and SF-36.

The ANCOVA model for repeated measures applied to the changes versus baseline at the end of treatment and at the end of follow-up, applied to general well-being, IBS-QoL and SF-36 data, did not show statistically significant difference between treatments.

IBS-QoL scores were characterised by high variability. Except from the sexual score that remained unchanged, all other items at the end of follow-up versus baseline showed higher values in the mesalazine group than in the placebo group, although there 


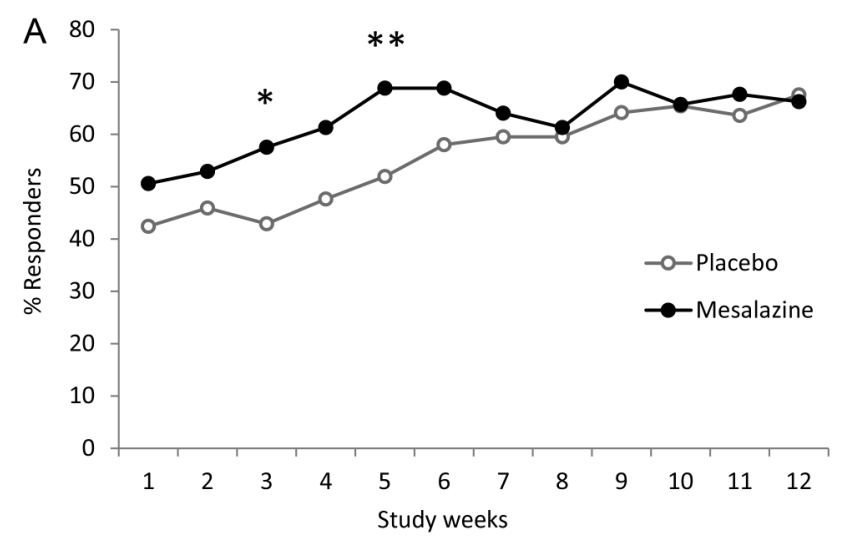

B

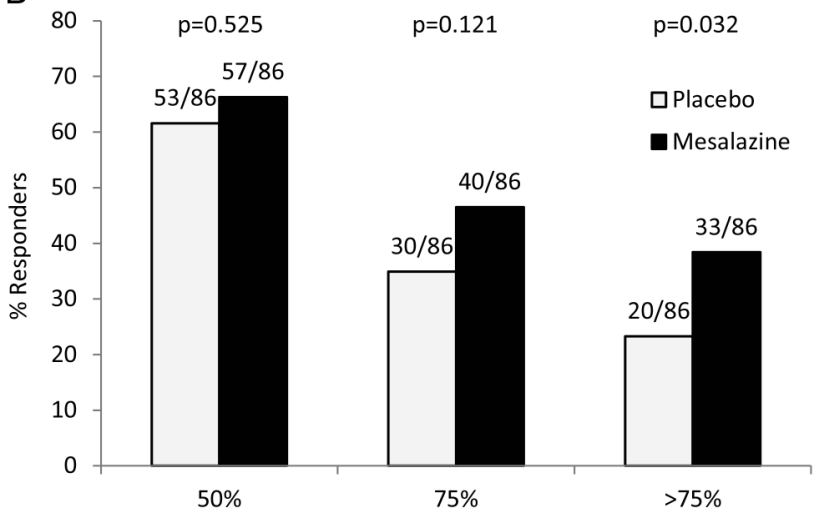

Figure 4 Secondary efficacy analysis (satisfactory relief of the overall IBS symptoms). The logistic model for repeated measures did not reveal statistically significant effect for treatment $(p=0.155)$ nor interaction between treatment and time $(p=0.640)$. The answers varied significantly with time $(p=0.004)$. Using the same model to test the simple main effect, a borderline significant effect was detected at week $3(p=0.060)$ and a statistical significance was detected at week 5 $(p=0.038)(A)$. According to the prevalence approach, responder patients were $66.3 \%$ in the mesalazine group versus $61.6 \%$ in the placebo group, with a $\delta$ in favour of the mesalazine group of $4.7 \%$ $(\mathrm{p}=0.525 ; 95 \% \mathrm{Cl}-9.7 \%$ to $19.0 \%)$ (B). Explorative analyses. With the $75 \%$ rule, $46.5 \%$ of patients in the mesalazine group were responders versus $34.9 \%$ in the placebo group, with a $\delta$ difference of $11.6 \%$ $(\mathrm{p}=0.121 ; 95 \% \mathrm{Cl}-3.0 \%$ to $26.2 \%$ ) (B). With the $>75 \%$ rule, $38.4 \%$ of responder patients were identified in the mesalazine group versus $23.3 \%$ of patients in the placebo group, with a $\delta$ difference of $15.4 \%$ ( $\mathrm{p}=0.032 ; 95 \% \mathrm{Cl} 1.5 \%$ to $28.7 \%)$ (B).

were no significant differences between the two groups (data not shown).

SF-36 scores were characterised by high variability. Except from the role limitations due to emotional problems score, the vitality score, the social functioning score and the global mental score, all other items at the end of follow-up versus baseline showed higher values in the mesalazine group than in the placebo group, although there were no significant differences between the two groups (data not shown).

The average overall satisfaction with treatment was $6.4 \pm 2.5$ in the mesalazine group and $5.8 \pm 2.7$ in the placebo group $(\mathrm{p}=0.266)$.

\section{Rescue medication}

In the placebo group, the average frequency of rescue medication was higher than that of the mesalazine group in most of the weeks of observation, but the differences were not statistically significant. In the ANCOVA model for repeated measures on the weekly rescue medication frequencies, neither treatment effect $(p=0.482)$ nor interaction treatment week effect $(p=0.492)$ was significant.

\section{Safety}

The safety profile of mesalazine was similar to that of placebo (table 3). Serious treatment emergent adverse events (ie, occurring after the first study medication exposure) were reported in four patients in the mesalazine group versus zero patients in the placebo groups and included two cases of gastroenteritis, one case of ischaemic colitis and one case of relapse of breast cancer. No deaths occurred during the study.

\section{DISCUSSION}

This multicentre controlled trial assessed the efficacy and safety of mesalazine in the treatment of patients with IBS. The percentage of patients giving affirmative answers to the weekly question on satisfactory relief of abdominal pain or discomfort for $>50 \%$ of the treatment period was set as the primary endpoint of the study. With this cut-off value, compared with placebo, mesalazine did not increase the percentage of responders.

There are several reasons explaining why we chose as primary endpoint the satisfactory relief of abdominal pain or discomfort for at least $50 \%$ of weeks over a 3 -month treatment period. First, adequate or satisfactory relief of abdominal pain or overall IBS symptoms were the standards for primary outcome assessment in IBS trials at the time of the study design. ${ }^{26}$ Responders were considered to be those patients who reported adequate or satisfactory relief in at least $50 \%$ of weeks over the treatment period. $^{27} 28$ These binary endpoints were used in several trials in IBS. ${ }^{27}{ }^{29}$ Abdominal pain is the key symptom of IBS and correlates with the severity of disease $\mathrm{e}^{30}$ and the use of healthcare resources. ${ }^{31}$ Second, in our previous proof-of-concept study assessing the effect of mesalazine on intestinal immune cells, we observed a promising effect of mesalazine on abdominal pain. ${ }^{19}$

The new Food and Drug Administration and European Medicines Agency recommended end points substantially different from the past, ${ }^{32}$ making the comparison between old and new studies complex. Based on previous studies showing a similar degree of immune cell activation both in patients with IBS-D and IBS-C, ${ }^{33}$ we hypothesised that mesalazine could be effective in the reduction of abdominal pain in all subgroups of patients with IBS. In line with this hypothesis, we did not show any significant difference in treatment responses based on bowel habits.

One of the possible explanations for the lack of effect of mesalazine using the 50\% cut-off value could be the high placebo response seen in this trial, which likely masked drug efficacy. The high number of planned visits, a short run-in period, the selection of patients from tertiary centres and the type of primary endpoint chosen could have all played a role in the high placebo response recorded in the present study, ${ }^{34}$ and generally, in the high level of responders. Furthermore, the enrolment of both male and female subjects and patients with mild symptoms, due to the lack of a minimum requirement for the severity of abdominal discomfort/pain or duration of disease in the inclusion criteria, may have contributed to the high placebo response. Another limitation of the study is related to the small sample size. Data indicating that mesalazine had positive effects but did not reach a statistical significant suggest a type 2 error. Our sample size calculation may have been limited by lack of data from previous placebo-controlled trials assessing the efficacy of mesalazine in IBS, with the exception of our previous proof-of-concept study. ${ }^{19}$ 
Table 2 Descriptive statistics by treatment group and by week for abdominal pain/discomfort, bloating, general well-being, IBS-QoL and SF-36

\begin{tabular}{|c|c|c|c|c|}
\hline & & Mesalazine $(n=86)$ & Placebo $(n=86)$ & $\mathrm{p}$ Value \\
\hline \multicolumn{5}{|l|}{ Abdominal pain/discomfort intensity } \\
\hline Mean (SD) & Baseline (week 2) & $4.59(2.54)$ & $4.50(2.34)$ & \\
\hline Mean change versus baseline (SD) & $\begin{array}{l}\text { End of treatment } \\
\text { End of follow-up }\end{array}$ & $\begin{array}{l}-1.07(2.54) \\
-1.07(2.63)\end{array}$ & $\begin{array}{l}-1.21(2.36) \\
-0.85(2.94)\end{array}$ & \\
\hline Least squares means $(\mathrm{SE})^{* * *}$ & & $1384.18(63.80)$ & $1394.80(58.80)$ & $0.903^{*}$ \\
\hline \multicolumn{5}{|l|}{ Bloating intensity } \\
\hline Mean (SD) & Baseline (week 2) & $5.37(2.77)$ & $4.97(2.65)$ & \\
\hline Mean change versus baseline (SD) & $\begin{array}{l}\text { End of treatment } \\
\text { End of follow-up }\end{array}$ & $\begin{array}{l}-1.30(2.63) \\
-0.93(2.35)\end{array}$ & $\begin{array}{l}-0.88(2.54) \\
-1.25(3.15)\end{array}$ & \\
\hline Least squares means $(\mathrm{SE})^{\star \star *}$ & & $1305.12(62.41)$ & $1418.13(57.06)$ & $0.184^{*}$ \\
\hline \multicolumn{5}{|l|}{ General well-being } \\
\hline Mean (SD) & Baseline (week 2) & $4.75(2.45)$ & $4.11(2.50)$ & \\
\hline Mean change versus baseline (SD) & $\begin{array}{l}\text { End of treatment } \\
\text { End of follow-up }\end{array}$ & $\begin{array}{l}0.53(2.50) \\
0.39(2.68)\end{array}$ & $\begin{array}{l}1.31(2.84) \\
1.43(3.87)\end{array}$ & \\
\hline Least squares means $(\mathrm{SE})^{* * *}$ & & $1283.61(61.55)$ & $1314.83(57.24)$ & $0.712^{*}$ \\
\hline \multicolumn{5}{|l|}{ IBS-QoL total score } \\
\hline Mean (SD) & Baseline & $62.93(17.36)$ & $65.16(16.41)$ & \\
\hline Mean change versus baseline (SD) & $\begin{array}{l}\text { End of treatment } \\
\text { End of follow-up }\end{array}$ & $\begin{array}{l}7.06(12.89) \\
9.34(13.28)\end{array}$ & $\begin{array}{l}5.81(11.60) \\
6.52(14.30)\end{array}$ & \\
\hline Least squares means $(\mathrm{SE})^{*}$ & & $7.89(1.41)$ & $6.12(1.40)$ & $0.374^{* *}$ \\
\hline \multicolumn{5}{|l|}{ SF-36 total score } \\
\hline Mean (SD) & Baseline & $62.68(16.01)$ & $65.09(15.38)$ & \\
\hline Mean change versus baseline (SD) & $\begin{array}{l}\text { End of treatment } \\
\text { End of follow-up }\end{array}$ & $\begin{array}{l}5.78(12.90) \\
8.17(14.27)\end{array}$ & $\begin{array}{l}5.74(13.44) \\
7.60(15.63)\end{array}$ & \\
\hline Least squares means $(\mathrm{SE})^{* *}$ & & $125.98(7.37)$ & $125.19(7.35)$ & $0.940^{* * *}$ \\
\hline
\end{tabular}

By increasing the percentage of time required to define the responders (75 and $>75 \%$ rule), we found markedly higher response rates in the mesalazine group compared with the placebo group. This effect was evident in the satisfactory relief of abdominal pain as well as in the overall IBS symptom endpoints. In particular, a statistically significant improvement in the satisfactory relief of the overall IBS symptoms was found with the $>75 \%$ rule. The $\delta$ difference over placebo was $>15 \%$, and the number needed to treat was 7 . The $>75 \%$ rule is a more restrictive outcome measure and selects a smaller

Table 3 Treatment-emergent adverse events during the study

\begin{tabular}{lcc}
\hline Event & Placebo $(\mathbf{n}=91)$ & Mesalazine $(\mathbf{n}=88)$ \\
\hline Adverse events & & \\
$\quad$ Upper respiratory tract infections & $7(7.7 \%)$ & $11(12.5 \%)$ \\
Diarrhoea & $10(11.0 \%)$ & $6(6.8 \%)$ \\
Headache & $8(8.8 \%)$ & $5(5.7 \%)$ \\
Nausea & $2(2.2 \%)$ & $4(4.6 \%)$ \\
Flatulence & $4(4.4 \%)$ & $3(3.4 \%)$ \\
Vomiting & $3(3.3 \%)$ & $2(2.3 \%)$ \\
Dyspepsia & $4(4.4 \%)$ & $0(0 \%)$ \\
Serious adverse events & & \\
Gastroenteritis & $0(0 \%)$ & $2(2.3 \%)$ \\
Ischaemic colitis & $0(0 \%)$ & $1(1.1 \%)$ \\
Breast cancer & $0(0 \%)$ & $1(1.1 \%)$ \\
\hline
\end{tabular}

Adverse events are listed in descending order of frequency in the mesalazine group. The adverse events listed were reported in $2 \%$ or more of the patients in either treatment group. percentage of responders. These data indicate that a subgroup of patients with IBS responded better to mesalazine than placebo. These more restrictive outcome measures have been previously used in the tegaserod ${ }^{28}$ and linaclotide ${ }^{35}$ trials and help to distinguish the 'sustained' responders to the active treatment from those responding to placebo. In line with this observation, in two randomised trials on linaclotide for chronic constipation, only $5 \%$ of subjects who received placebo was considered responders due to the rigour of the primary endpoint. ${ }^{35}$ However, due to the explorative nature of these analyses, further studies are needed to confirm these results.

In addition to improving overall IBS symptoms with the $75 \%$ rule, mesalazine improved the percentage of responders to pain and discomfort at week 8 (50\% rule) and the percentage responders to overall symptoms at weeks 3 and 5 (50\% rule). There was also a trend in a better improvement of pain intensity and bloating as well as quality of life and a lower requirement of rescue medications with mesalazine, although these data did not reach statistical significance.

Although a subgroup of patients was more responsive to the mesalazine treatment, our data did not allow to identify these subjects. Patients with low-grade mucosal inflammation are possible candidates for a good response. In previous studies, we showed that low-grade intestinal inflammation is present roughly in half of the IBS population. ${ }^{336}$ However, in the present study, we did not preselect these patients as the requirement of invasive techniques would have significantly reduced the enrolment rate.

Similar percentages of patients in the mesalazine group and in the placebo group had adverse events. In addition, the favourable safety profile of mesalazine was demonstrated in long-term 
studies in the maintenance of remission of UC. ${ }^{37}$ Although the study showed no major adverse events in the mesalazine group as compared with placebo, long-term and larger studies are needed to assess this issue in patients with IBS.

In conclusion, the results of the present study indicate that a number of patients show a sustained therapy response and benefits from mesalazine therapy. Larger studies assessing the clinical predictors and confirming the beneficial effects of mesalazine are now required.

\section{Author affiliations}

${ }^{1}$ Department of Medical and Surgical Sciences, Centre for Applied Biomedical Research, University of Bologna, Bologna, Italy

${ }^{2}$ Division of Gastroenterology SOD2, University Hospital Careggi, Florence, Italy

${ }^{3}$ Gastroenterology Unit, Ospedale Maggiore, Policlinico, Milan, Italy

${ }^{4}$ Gastroenterology Unit, Department of Medical and Surgical Sciences, University of Bologna, Bologna, Italy

${ }^{5}$ Gastroenterology Unit, Department of Gastroenterology, University of Pisa, Pisa, Italy

${ }^{6}$ Department of Gastroenterology and Hepatology, Università Politecnica delle Marche, Ancona, Italy

${ }^{7}$ Gastroenterology Unit, University of Verona, Verona, Italy

${ }^{8}$ Division of Gastroenterology, Casa Sollievo Sofferenza Hospital, IRCCS, San Giovanni Rotondo, Italy

${ }^{9}$ Gastroenterology Unit of Comacchio/Lagosanto, Ferrara, Italy

${ }^{10}$ Gastroenterology Unit, University Campus Bio-Medico of Rome, Rome, Italy

${ }^{11}$ Digestive Motility Diseases, Department of Clinical Medicine and Surgery, Federico II University Hospital, Naples, Italy

${ }^{12}$ Gastroenterology Unit, S. Martino Hospital, Belluno, Italy

${ }^{13}$ Gastroenterology Unit, Department of Clinical Science, "L. Sacco" University Hospital, Milan, Italy

${ }^{14}$ Department of Medicine and Aging Sciences and CESI, G. D'Annunzio University and Foundation, Chieti, Italy

${ }^{15}$ Gastroenterology Unit, St. Andrea Hospital, Vercelli, Italy

${ }^{16}$ Department of Digestive Diseases, Hepatology and Clinical Nutrition, Dell'Angelo Hospital, Venice, Italy

${ }^{17}$ Division of Gastroenterology, AO San Camillo Forlanini, Rome, Italy

${ }^{18} \mathrm{G}$ astroenterology and Digestive Endoscopy Unit, IRCCS Policlinico San Donato, San Donato Milanese, Italy

${ }^{19}$ Santissima Annunziata Hospital, Cento, Italy

Acknowledgements We are grateful to Gabriella Bartesaghi from SOFAR S.p.A and to Daniela Garavaglia and Aldo Poli from OPIS for their constant support in all phases of the study and assistance in the preparation of the manuscript. We would like to acknowledge the contribution of the following study participants during the trial: Angelo Andriulli, Rosanna Cogliandro, Lara Bellacosa, Giovanni Carini, Maria Raffaella Barbaro.

Contributors GB, CC, RC and VS planned the study, designed the protocol, contributed to the writing of the manuscript and to the screening and periodic visits of the patients. $V A, G B, F B, M B, A B, L B, F B, P B, M C, R C, B G, M L S, P M, M N, M R$, $A S, M L S, M V$ and $G Z$ contributed to the screening and periodic visits of the patients. All authors have access to the study data and have reviewed and approved the final manuscript.

Funding This study was supported by Sofar S.p.A., Trezzano Rosa, Milan, Italy. The funding agency had no role in the study design, collection, analysis, data interpretation or writing of the report.

Competing interests GB has been a consultant, served on the advisory board, received speaker's bureau fees, honorarium and travel support to meetings related to this study from the study sponsor, and has received consultancy fees and/or speaker fees from Danone, Yakult, Ironwood, Malesci, Nestlè, Noos, Synergy, Alfa Wassermann, Almirall and Shire. CC received research funding and travel support to meetings from the study sponsor. VA has been a consultant/speaker, served on the advisory board or received research grants fromG iuliani, Abbvie, MS\&D, Ferring, Takeda, Nycomed, Shire, Cosmo Pharmaceuticals, Sofar, SEDA, Hospira, Otsuka, Astra Zeneca, Dicofarm, Alfa Wassermann, Johnson \& Johnson. FB has received research funding, travel support to meetings and speaker fees from Alfa Wasserman, Aptalis, Astra Zeneca, Sofar. MR received travel support to meetings from the study sponsor. VS has received consultancy fees and/or speaker fees from Alfa Wassermann, Almirall, Angelini Aptalis, CM\&D Pharma, Farmaderma, Ironwood, Italchimici, Janssen, Norgine, Shire, Takeda, Valeas, Vibrant and Zeria. The remaining authors disclose no conflicts.

Patient consent Obtained.

Ethics approval The protocol was approved by an independent ethics committee at each centre and carried out according to the Declaration of Helsinki and the principles of good clinical practice.
Provenance and peer review Not commissioned; externally peer reviewed.

Open Access This is an Open Access article distributed in accordance with the Creative Commons Attribution Non Commercial (CC BY-NC 4.0) license, which permits others to distribute, remix, adapt, build upon this work non-commercially, and license their derivative works on different terms, provided the original work is properly cited and the use is non-commercial. See: http://creativecommons.org/ licenses/by-nc/4.0/

\section{REFERENCES}

1 Longstreth GF, Thompson WG, Chey WD, et al. Functional bowel disorders. Gastroenterology 2006;130:1480-91.

2 Camilleri M. Peripheral mechanisms in irritable bowel syndrome. N Engl I Med 2013;368:578-9.

3 Barbara G, Cremon C, Carini G, et al. The immune system in irritable bowel syndrome. J Neurogastroenterol Motil 2011;17:349-59.

4 Spiller R, Garsed K. Postinfectious irritable bowel syndrome. Gastroenterology 2009;136:1979-88.

5 Cremon C, Stanghellini V, Pallotti F, et al. Salmonella gastroenteritis during childhood is a risk factor for irritable bowel syndrome in adulthood. Gastroenterology 2014;147:69-77.

6 Piche T, Barbara G, Aubert P, et al. Impaired intestinal barrier integrity in the colon of patients with irritable bowel syndrome: involvement of soluble mediators. Gut 2009;58:196-201.

7 Cremon C, Carini G, Wang B, et al. Intestinal serotonin release, sensory neuron activation, and abdominal pain in irritable bowel syndrome. Am J Gastroenterol 2011;106:1290-8.

8 Foley S, Garsed K, Singh G, et al. Impaired uptake of serotonin by platelets from patients with irritable bowel syndrome correlates with duodenal immune activation. Gastroenterology 2011;140:1434-43 e1.

9 Barbara G, Wang B, Stanghellini V, et al. Mast cell-dependent excitation of visceral-nociceptive sensory neurons in irritable bowel syndrome. Gastroenterology 2007;132:26-37.

10 Cenac N, Andrews CN, Holzhausen M, et al. Role for protease activity in visceral pain in irritable bowel syndrome. J Clin Invest 2007;117:636-47.

11 Buhner S, Li Q, Berger T, et al. Submucous rather than myenteric neurons are activated by mucosal biopsy supernatants from irritable bowel syndrome patients. Neurogastroenterol Motil 2012;24:1134-e572.

12 Buhner S, Li Q, Vignali S, et al. Activation of human enteric neurons by supernatants of colonic biopsy specimens from patients with irritable bowel syndrome. Gastroenterology 2009;137:1425-34.

13 Balestra B, Vicini R, Cremon C, et al. Colonic mucosal mediators from patients with irritable bowel syndrome excite enteric cholinergic motor neurons. Neurogastroenterol Motil 2012;24:1118-e570.

14 Zucchelli M, Camilleri M, Andreasson AN, et al. Association of TNFSF15 polymorphism with irritable bowel syndrome. Gut 2011;60:1671-7.

15 Swan C, Duroudier NP, Campbell E, et al. Identifying and testing candidate genetic polymorphisms in the irritable bowel syndrome (IBS): association with TNFSF15 and TNFalpha. Gut 2013;62:985-94.

16 Piche T, Saint-Paul MC, Dainese R, et al. Mast cells and cellularity of the colonic mucosa correlated with fatigue and depression in irritable bowel syndrome. Gut 2008;57:468-73.

17 Vivinus-Nebot M, Dainese R, Anty R, et al. Combination of allergic factors can worsen diarrheic irritable bowel syndrome: role of barrier defects and mast cells. Am I Gastroenterol 2012;107:75-81.

18 Simren M, Barbara G, Flint HJ, et al. Intestinal microbiota in functional bowel disorders: a Rome foundation report. Gut 2013:62:159-76.

19 Corinaldesi R, Stanghellini V, Cremon C, et al. Effect of mesalazine on mucosal immune biomarkers in irritable bowel syndrome: a randomized controlled proof-of-concept study. Aliment Pharmacol Ther 2009;30:245-52.

20 Dunlop SP, Jenkins D, Neal KR, et al. Randomized, double-blind, placebo-controlled trial of prednisolone in post-infectious irritable bowel syndrome. Aliment Pharmacol Ther 2003;18:77-84.

21 Barbara G, Stanghellini V, Cremon C, et al. Aminosalicylates and other anti-inflammatory compounds for irritable bowel syndrome. Dig Dis 2009;27(Suppl 1):115-21.

22 Klooker TK, Braak B, Koopman KE, et al. The mast cell stabiliser ketotifen decreases visceral hypersensitivity and improves intestinal symptoms in patients with irritable bowel syndrome. Gut 2010;59:1213-21.

23 Mowat $C$, Cole A, Windsor A, et al. Guidelines for the management of inflammatory bowel disease in adults. Gut 2011;60:571-607.

24 Patrick DL, Drossman DA, Frederick IO, et al. Quality of life in persons with irritable bowel syndrome: development and validation of a new measure. Dig Dis Sci 1998:43:400-11.

25 Ware JE Jr, Sherbourne CD. The MOS 36-item short-form health survey (SF-36). I. Conceptual framework and item selection. Med Care 1992;30:473-83.

26 Irvine EJ, Whitehead WE, Chey WD, et al. Design of treatment trials for functional gastrointestinal disorders. Gastroenterology 2006;130:1538-51. 
27 Camilleri $M$, Northcutt AR, Kong $S$, et al. Efficacy and safety of alosetron in women with irritable bowel syndrome: a randomised, placebo-controlled trial. Lancet 2000;355:1035-40.

28 Tack J, Muller-Lissner S, Bytzer P, et al. A randomised controlled trial assessing the efficacy and safety of repeated tegaserod therapy in women with irritable bowel syndrome with constipation. Gut 2005:54:1707-13.

29 Muller-Lissner SA, Fumagalli I, Bardhan KD, et al. Tegaserod, a 5-HT(4) receptor partial agonist, relieves symptoms in irritable bowel syndrome patients with abdominal pain, bloating and constipation. Aliment Pharmacol Ther 2001;15:1655-66.

30 Lembo A, Ameen VZ, Drossman DA. Irritable bowel syndrome: toward an understanding of severity. Clin Gastroenterol Hepatol 2005;3:717-25.

31 Spiegel $B$, Bolus $R$, Harris $L A$, et al. Measuring irritable bowel syndrome patient-reported outcomes with an abdominal pain numeric rating scale. Aliment Pharmacol Ther 2009;30:1159-70.
32 Corsetti M, Tack J. FDA and EMA end points: which outcome end points should we use in clinical trials in patients with irritable bowel syndrome? Neurogastroenterol Motil 2013;25:453-7.

33 Cremon C, Gargano L, Morselli-Labate AM, et al. Mucosal immune activation in irritable bowel syndrome: gender-dependence and association with digestive symptoms. Am J Gastroenterol 2009;104:392-400.

34 Enck P, Bingel U, Schedlowski M, et al. The placebo response in medicine: minimize, maximize or personalize? Nat Rev Drug Discov 2013:12:191-204

35 Lembo AJ, Schneier HA, Shiff SJ, et al. Two randomized trials of linaclotide for chronic constipation. N Eng/ J Med 2011;365:527-36.

36 Barbara G, Stanghellini V, De Giorgio R, et al. Activated mast cells in proximity to colonic nerves correlate with abdominal pain in irritable bowel syndrome. Gastroenterology 2004;126:693-702.

37 Feagan BG, Macdonald JK. Oral 5-aminosalicylic acid for maintenance of remission in ulcerative colitis. Cochrane Database Syst Rev 2012;10:CD000544. 